\title{
Canadian Society for Vascular Surgery consensus statement on endovascular aneurysm repair
}

A bdominal aortic aneurysm (AAA) occurs in $5 \%$ of men and $1 \%$ of women over the age of 65 . AAA rupture is fatal in $80 \%-90 \%$ of cases when prehospital deaths are included. Thus, elective repair should be considered for any AAA with a maximal diameter of $5.5 \mathrm{~cm}$ (men) or $5.0 \mathrm{~cm}$ (women). ${ }^{1}$ Traditional elective surgical repair carries a $4 \%-5 \%$ risk of death. Factors such as increased age and cardiac, respiratory and renal comorbidities can double or triple the risk of perioperative morbidity and death.

Endovascular AAA repair (EVAR) is a minimally invasive catheter-based procedure that offers a viable alternative to open surgical repair. Collapsed grafts (composed of fabric and stents) are introduced over guide wires though small incisions in the femoral arteries, positioned in the aorta under $\mathrm{x}$-ray guidance, and fixed in place with balloon catheters. These devices are held in place by the radial force of the stents and by hooks or barbs that anchor into the aortic wall. Selection of patients with vascular anatomy that meets established criteria improves the chance of complete aneurysm exclusion. For such patients, EVAR reduces the major risks associated with open AAA repair. As is the case with all new surgical procedures, additional training and adequate case volumes are necessary to achieve and maintain expertise.

Since the first case report in 1991, EVAR stent-grafts have evolved from homemade prototypes to commercially manufactured products evaluated in mul+ tiple trials. The US Food and Drug Administration initially approved 2 commercial stentgrafts in 1999, and approvals have followed in Canada.

In contrast to open surgery, EVAR does not require an abdominal incision or dissection and clamping of the aorta. It also avoids significant lowertorso ischemia, minimizes blood loss and reduces the mortality associated with repair as well as the incidence of moderate to severe systemic and local complications. $^{2-4}$ ICU and hospital stays are reduced by more than $50 \% .{ }^{4}$ EVAR allows a more rapid return to normal activity than open repair, which can require 3-4 months of recovery time. Although complications are significantly reduced for those who undergo EVAR, technical failures do occur (e.g., incomplete aneurysm exclusion due to "endoleaks," residual blood leaking into the remaining sac). In one trial, conversion to open repair occurred in only $1.9 \%$ of cases. ${ }^{3}$ Lifelong followup is required to monitor the patient for endoleaks, aneurysm enlargement and device migration or failure.

There is an increasing trend toward EVAR for elective AAA repair. ${ }^{2}$ In New York State, EVAR was performed for over $50 \%$ of aneurysm patients in 2003. Despite a higher incidence of comorbid conditions in the EVAR group, the mortality associated with open repair was $4.2 \%$, compared with $0.8 \%$ for EVAR. The US National Inpatient Sample for $2001 \mathrm{docu}-$ mented that the EVAR group had fewer complications and deaths $(1.3 \%$ v. $3.8 \%)$ and shorter hospital stays compared with the open repair group.

Two randomized prospective trials have recently been published comparing EVAR with open surgery in patients who were healthy enough for open AAA repair. In a study involving 1082 patients, the 30 -day mortality was $1.7 \%$ after EVAR versus $4.7 \%$ after open repair. ${ }^{3}$ The patients who had undergone EVAR required more secondary interventions $(9.8 \%$ v. $5.8 \%)$; however, most of these were percutaneous radiological procedures. A smaller study found a similar reduction in operative mortality when EVAR was compared with open surgery. ${ }^{4}$ The incidence of moderate and severe systemic complications was significantly reduced with EVAR (11.7\% v. 26.6\% for open repair), as was blood loss, incidence of transfusion, and ICU and hospital stay. The mortality benefit is magnified in those judged to be at high risk for open repair: $4.7 \%$ in patients undergoing EVAR compared with $19.2 \%$ for open repair. ${ }^{6}$ Long-term durability and success is being achieved, although re-intervention rates after EVAR are higher than after conventional surgery.?

\section{Summary}

EVAR is a minimally invasive approach to AAA repair that lowers operative complication rates and mortality when compared with open surgery. At present, EVAR requires lifelong follow-up because of potential late graft failure; however, with appropriate follow-up the risk of graft failure leading to aneurysm rupture is very low. Internationally, EVAR has become a standard of care for AAA patients with suitable vessel anatomy. Although the device costs exceed those of open repair, at US\$22 862 per procedure the cost-effectiveness of EVAR compares favourably with other life-saving technologies such as coronary artery bypass grafting for left main coronary artery disease (\$9500) and dialysis (\$54 400 per year). ${ }^{8}$ EVAR is continuing to evolve with experience and technologic advances. Devices are being developed and used to improve outcomes for patients with ruptured AAAs and for thoracic, thoracoabdominal and perirenal aneurysms. 


\section{Analysis}

Canadian Society for Vascular Surgery recommendations

EVAR is now an accepted part of vascular surgical practice at referral centres. The Canadian Society for Vascular Surgery (CSVS) does not advocate an adjustment to the current thresholds established in the literature for elective aneurysm repair. In the management of an individual patient, all modalities of aneurysm therapy should be discussed before a treatment is selected.

1. The CSVS recommends that EVAR should be the procedure of choice for patients with suitable vascular anatomy who are at intermediate or high risk $(6 \%-10 \%)$ for perioperative morbidity or death with open repair.

2. For patients at low risk $(2 \%-4 \%)$, open repair remains the current standard. For those with suitable vascular anatomy for EVAR, the final decision should also take into account the patient's wishes. Longer term outcome data are required before EVAR can replace open repair as the treatment of choice for low-risk patients.

3. EVAR procedures require specialized training and co- operation between specialists with complementary areas of expertise. They should be performed in centres experienced with aneurysm repair and with sufficient EVAR volume to enable appropriate data collection and auditing of results.

4. Appropriate training in endovascular therapies and interventional procedures is required for vascular surgery trainees. Training programs are needed for existing vascular surgeons and interventional radiologists currently in practice to allow this procedure to be safely implemented and disseminated across the country.

Thomas F. Lindsay, on behalf of the Canadian Society for Vascular Surgery

Division of Vascular Surgery

Toronto General Hospital /

University Health Network

Toronto General Hospital

Toronto, Ont.

Competing interests: Dr. Lindsay has received travel assistance to attend an interdisciplinary meeting about EVAR sponsored by W.L. Gore \& Associates.

\section{References}

1. Lederle FA, Wilson SE, Johnson GR, Reinke DB, Littooy FN, Acher CW, et al. Immediate repair compared with surveillance of small abdominal aortic aneurysms. N Engl 7 Med 2002; 346:1437-44

2. Anderson PL, Arons RR, Moskowitz AJ, Gelijns A, Magnell C, Faries PL, et al. A statewide experience with endovascular abdominal aortic aneurysm repair: rapid diffusion with excellent early results. 7 Vasc Surg 2004;39: 10-9.

3. Greenhalgh RM, Brown LC, Kwong GP, Powell JT, Thompson SG. Comparison of endovascular aneurysm repair with open repair in patients with abdominal aortic aneurysm (EVAR trial 1), 30-day operative mortality results: randomised controlled trial. Lancet 2004;364:843-8.

4. Prinssen M, Verhoeven EL, Buth J Cuypers PW, van Sambeek MR, Balm R, et al. A randomized trial comparing conventional and endovascular repair of abdominal aortic aneurysms. N Engl 7 Med 2004;351: 1607-18.

5. Lee WA, Carter JW, Upchurch G Seeger JM, Huber TS. Perioperative outcomes after open and endovascular repair of intact abdominal aortic aneurysms in the United States during 2001. 7 Vasc Surg 2004;39:491-6.

6. Teufelsbauer H, Prusa AM, Wolff K, Polterauer P, Nanobashvili J, Prager $M$, et al. Endovascular stent grafting versus open surgical operation in patients with infrarenal aortic aneurysms: a propensity scoreadjusted analysis. Circulation 2002; 106:782-7.

7. Cao P, Verzini F, Parlani G, Romano L, De Rango P, Pagliuca V, et al. Clinical effect of abdominal aortic aneurysm endografting: 7-year concurrent comparison with open repair. 7 Vasc Surg 2004;40:841-8.

8. Patel ST, Haser PB, Bush HL Jr, Kent KC. The cost-effectiveness of endovascular repair versus open surgical repair of abdominal aortic aneurysms: A decision analysis model. 7 Vasc Surg 1999;29(6):958-72.

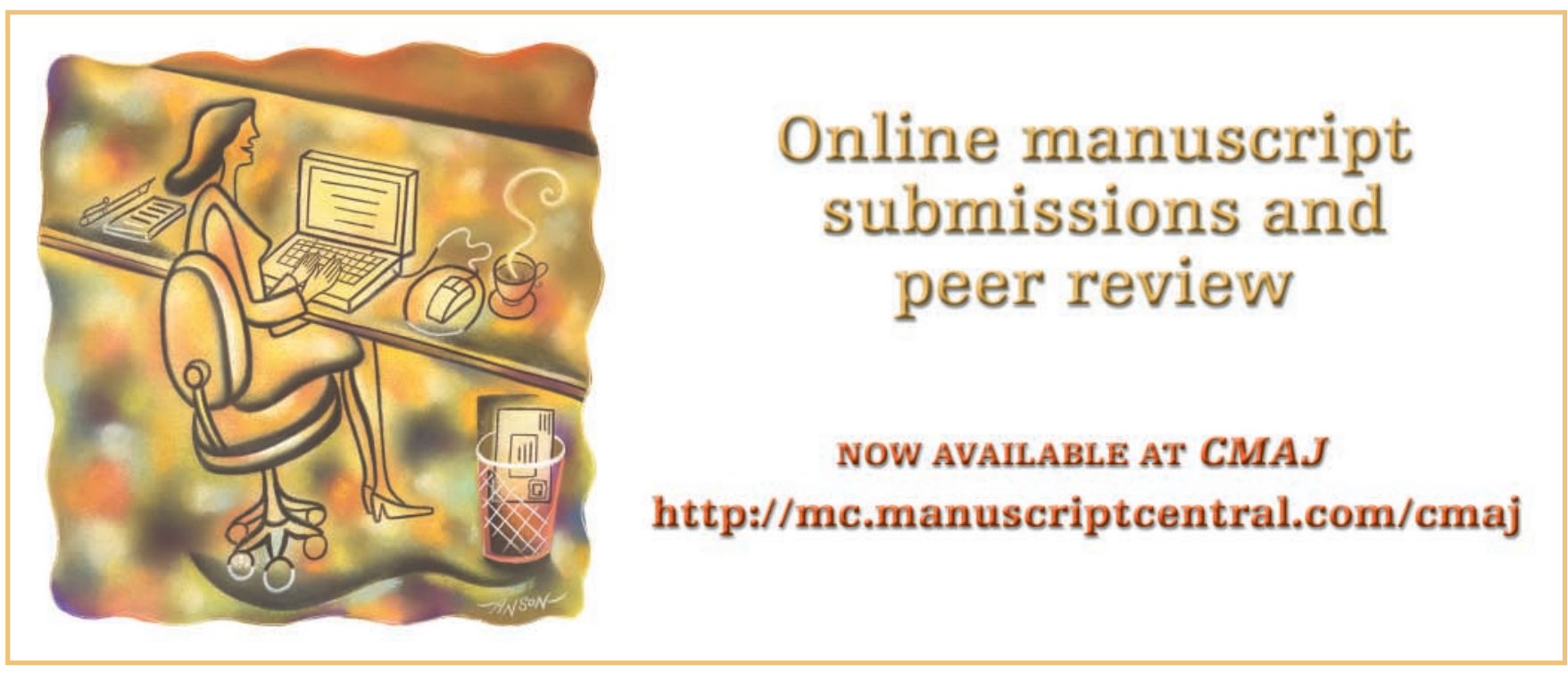

